\title{
ALGUNAS CONSIDERACIONES SOBRE LA GRAMÀTICA Y SUS IMPLICACIONES PARA LA ENSEÑANZA APRENDIZAJE DE LA PRODUCCIÒN ESCRITA
}

Olga Espinal*

RESUMEN

Este artículo presenta una reflexión acerca de la gramática textual y de la lengua aplicadas a la enseñanza del español como lengua materna. En ese sentido, se estudia la gramática textual como una parte de la disciplina llamada Lingüistica del texto que se ocupa de describir y explicar los patrones recurrentes en el uso real de la lengua y en el contexto en que este uso se produce.

La lingüistica textual ha aportado una serie de conceptos que nos permiten integrar los aspectos formales tratados en las gramáticas tradicional y estructural, a las que podríamos denominar genéricamente como gramática de la lengua. Uno de esos conceptos es la cohesión que se refiere a relaciones significativas entre las oraciones y los elementos que posibilitan dicha relación.

En cuanto a la gramática de la lengua, ésta ha descrito aspectos formales: sintácticos, morfológicos y ortográficos que permiten la unificación de la lengua escrita. Esta unificación permite una comunicación eficaz entre los usuarios de una misma lengua. No hay sentido sin forma: la unidad indivisible de estos dos elementos constituye el significado. Esto se ilustra con los ejemplos de las producciones escritas de los estudiantes.

Se plantean unas recomendaciones finales para los maestros de lengua.

PALABRAS CLAVES:

Gramática textual, gramática de la lengua, superestructura, macroestructura, frase nominal, referente, cohesión, coherencia, enseñanza de la lengua, concordancia, ortografía

(") Area de Humanidades - INTEC 


\section{INTRODUCCIÓN}

El surgimiento de investigaciones y reflexiones teóricas orientadas a la comunicación, es decir, al estudio no sólo de las emisiones lingüística completas, sino también del contexto en que éstas son emitidas, da lugar a la disciplina llamada lingüística del texto, cuyos principales exponentes son: Teun A. Van Dijk, Siegfred J. Schmidt, Ihwe y Petöfi. Esta disciplina, que se desarrolla al principio de los años setenta, constituye un avance dentro de los estudios lingüístico, puesto que no se limita a describir los aspectos verbales de las emisiones sino que se preocupa por el sentido de las oraciones y secuencias de oraciones de acuerdo con una intención comunicativa y una estructura textual.

Una noción de gran importancia en esta orientación es la de Gramática Textual, que se refiere a las propiedades lingüísticas de los textos, es decir, que va "más allá de la frontera de la oración" [1]. Para los gramáticos discursivos la gramática se define como los patrones recurrentes en el discurso real que lo configuran permanentemente. ${ }^{[2]}$

Van Dijk ha propuesto varios conceptos que sirven de base para una gramática textual, entre los que tenemos: la cohesión y la coherencia. La cohesión es una propiedad de carácter sintáctico. Se refiere a la conexión que ocurre entre las oraciones (o no) dentro de todo texto, con la finalidad de asegurar la interpretación de cada una de ellas, sus interrelaciones y, en definitiva, el sentido global del texto. La cohesión es lograda en los textos gracias a los enlaces o conectores, los signos de puntuación, las relaciones semánticas entre palabras (sinonimia, antonimia, hiponimia) o mediante la pronominalización.

La coherencia es una propiedad de carácter semántico. Se refiere a la forma en que el texto selecciona la información y la organiza, según sea relevante o no, dentro de la estructura comunicativa. 
Otro concepto interesante es el de superestructura textual, que ampliado por Josette Jolibert ${ }^{[3]}$, incluye:

- La silueta del texto, o sea la "distribución espacial" y los "bloques del texto" que lo conforman.

- La estructura esquemática, que es la forma global de un discurso [...] y las relaciones (jerárquicas) de sus respectivos fragmentos. Todos los tipos de texto tienen un "esquema global" que los caracteriza. Van Dijk asemeja ese esquema a la forma sintáctica de las oraciones pues también incluye unas "categorías" y a unas "reglas de formación". Por ejemplo, la superestructura del cuento incluye: una introducción, un nudo y un desenlace. ${ }^{[4]}$

Otro de los conceptos fundamentales de la lingüística del texto es la macroestructura, que está constituida, según el mismo Van Dijk, por las relaciones entre las proposiciones de un texto, es la "representación abstracta de la estructura global de su significado" [5]. Usando una terminología conocida por todos: es el tema o contenido general del texto. Determinar la macroestructura de un texto sirve para

“[...] reducir y organizar las grandes cantidades de información que tenemos que procesar (sea en la producción o en la comprensión) y buscamos hacerlo por medio de la construcción de sentidos, globalmente coherentes, de objetos, de relaciones entre objetos, de series de hechos, etc." [6]

Si aplicamos los principios de la gramática textual a la enseñanza de la comunicación escrita nos daremos cuenta que no bastan las reglas de la gramática de la lengua, la que adopta como unidad la oración y sus componentes, para lograr un discurso apropiado. Sin embargo, un uso adecuado de los recursos lingüísticos en el discurso implica el conocimiento de las reglas descritas por la gramática de la lengua. 
Si volvemos al concepto de cohesión nos daremos cuenta que éste se sitúa en el plano de la oración y en el plano del discurso. En el plano de la oración, aspectos como concordancia, orden de los constituyentes de la oración, uso de preposiciones y uso de pronombres ya han sido descritos por la gramática de la lengua. En el plano del discurso, se establece una relación entre las partes estructurales del discurso a través de las conjunciones o nexos.

Existe entonces la posibilidad de integrar los conceptos descritos por la gramática de la oración a una explicación de su uso a partir de los elementos extra e intra textuales que se manejan en la gramática textual.

\section{DIFICULTADES GRAMATICALES}

A continuación ejemplificaremos algunos errores que en la producción escrita de los estudiantes de la asignatura Lengua Castellana II en el trimestre Agosto-Octubre del año 2000, ${ }^{[7]}$ analizados y explicados a la luz de las gramáticas del texto y de la lengua:

\section{FUNCIÓN DE LAS PALABRAS SEGÚN EL REFERENTE ${ }^{[8]}$}

\subsection{Uso de la frase nominal plena, los pronombres y la anáfora cero}

La frase nominal plena es la oración en la que aparece el sustantivo que hace la función de sujeto en forma explícita. Algunos conceptos, relacionados con el uso de la frase nominal plena, son:

Referente: objeto, tema o individuo identificado en el texto. Se identifica con las preguntas: qué, quién, cuál. 
Predicado: contenido o cualidad del referente.

Elementos

Anafóricos: sustituyen al referente, cuando éste ya ha sido mencionado (pronombres, determinantes, artículos, sinónimos).

El referente, cuando está activo en la mente del lector-hablante, se puede sustituir por :

- Un pronombre

- La anáfora cero (sujeto omitido)

Veamos este texto correspondiente a una biografia:

"Debido (sic) que José era el primer hijo que tenía la pareja, el primer año se celebró con mucho alboroto, (sic) Tìos, tías, abuelos y primos fueron invitados a esa fiesta tan importante para sus padres $y$, claro, para José que disfrutó su cumpleaños. En ese primer año José residía en el sector del INVI"

Como se puede observar en el fragmento anterior, el referente es mencionado tres veces en un mismo párrafo cuando pudo haber sido sustituido por un elemento anafórico.

\subsection{Uso de los determinantes el y un}

Los determinantes son palabras que preceden al nombre y tienen la función de actualizarlo.

Se utiliza un cuando el referente no es identificable o no ha sido mencionado antes en el texto. Se utiliza el cuando el referente es identificable o ya ha sido mencionado en el texto.

En el ejemplo que presentaremos, el inicio de una narración de experiencias personales, se usa $\mathrm{el}$ sin haber mencionado antes al referente:

"Mi aventura se inició a las 7:45 P.M. cuando aborde (sic) el avión rumbo a mi destino anhelado, Italia, con (sic) escala en Francia" 


\section{Uso de anáfora cero y frase nominal según la actitud del hablante}

La frase nominal plena se utiliza en los textos según la actitud del hablante cuando el nivel de conocimiento y confianza con el referente es poco. La anáfora cero se usa cuando hay un nivel más cercano, de más confianza con el referente.

El ejemplo siguiente no evidencia este uso:

“(...) A las 4:30 salia a buscar a uno de mis amigos, Samuel, que es el único que vive lejos de todo.

Al llegar a casa de Samuel todo estaba (sic) color de rosa."

\section{EL ORDEN DE LAS PALABRAS SEGÚN LOS REFERENTES}

\subsection{La frase nominal plena vuelve a usarse cuando} termina o inicia una parte de la estructura textual: introducción, desarrollo o conclusión; pues el referente puede hacerse menos accesible.

\subsection{Uso de frases adverbiales que señalan limites en la estructura textual}

La biografia es un tipo de narración pues relata la vida de un personaje, generalmente en pasado.

Veamos algunos ejemplos del uso de los adverbios en las expresiones que señalan el desarrollo cronológico de la vida de Leonardo da Vinci ${ }^{[9]}$ :

Alli aprendió pintura y escultura y hacia 1473 colaboró con su maestro en su importante Bautismo de Cristo.

En 1481, cuando ya se había establecido por su cuenta,...

La caída del ducado de Milán en 1499 supuso para Leonardo da Vinci el final de una etapa especialmente satisfactoria de su carrera. 
Leonardo marchó en primer lugar a Mantua ...

.. finalmente regresó a Florencia...

Leonardo da Vinci volvió de nuevo a Milán,...

En este caso, la expresión anuncia cambio de referencia o tema:

La personalidad de Leonardo, sin embargo, supera ampliamente sus facetas Artísticas...

En el caso de los estudiantes, no utilizan nexos, ni frases adverbiales, solo verbos repetidos en el comienzo de párrafos distintos:

Todo empezó...

Al llegar a la casa...

Llegamos a la casa...

Al llegar a la casa...

Por último...

\section{LA GRAMÁTICA DE LA LENGUA}

Así como el acto de habla es la unidad de la comunicación, la oración es la unidad de la gramática como conjunto de reglas y normas. La oración se compone de sujeto y predicado y alrededor de estos dos componentes principales se organizan otros elementos que la conforman.

\subsection{Concordancia}

La concordancia es la correspondencia entre los verbos, sujetos, adjetivos, determinantes y otros componentes que pueda tener la oración.

Analicemos estas oraciones:

"Todos estaban muy contentos ya que este acontecimiento de que se juntara toda la familia y compartir entre primos es muy remoto ya que siempre falta alguien" 
Como podemos observar no existe una correspondencia entre los tiempos verbales el primer verbo (pasado) y el segundo verbo, referido al mismo hecho, en presente.

Otro ejemplo expresa:

“(...) Mi estadia (sic) fue de dos mes y medio, pero el tiempo fue (sic) tan rápido que se convirtieron en dos semanas"

\subsection{Preposiciones y conjunciones}

Son elementos que establecen conexiones entre las palabras lexicales (verbos, sustantivos, adjetivos...) de la oración. Para redactar un texto con sentido éste debe poseer las cualidades de coherencia y cohesión. Las conexiones dotan de cohesión a los textos.

Veamos este ejemplo:

"En casa de María esperamos el amanecer y en eso de las 8:00 nos fuimos para nuestra casa"

Las conjunciones relacionan palabras y oraciones en ${ }^{[10]}$ :

a) igualdad: $y, e, n i$

b) elección: $o, u, y a$, sea...

c) distribución: unas veces... otras veces...

d) expoliación: o sea, es decir...

e) continuidad: asi que, de modo que...

f) oposición: pero, mas, aunque, sin embargo...

En esta oración, la relación es de igualdad entre las proposiciones, sin embargo no se usa la conexión adecuada:

"Todos se divertian (sic) al maximo (sic), pero nadie estudiaba" 


\section{ORTOGRAFÍA}

Se considera que hay un uso adecuado de los recursos ortográficos cuando se escribe en correspondencia con las normas preestablecidas en la lengua en uso, en este caso la lengua española. Desde el punto de vista comunicativo, estas normas (de acentuación, de puntuación, de correspondencia sonido- grafia, de uso de las mayúsculas y abreviaturas) son importantes para establecer relaciones significativas dentro de un texto; tanto en oraciones como en secuencia de oraciones. En esta ocasión nos limitaremos a trabajar la puntuación y la acentuación.

\subsection{Puntuaciòn}

Los signos de puntuación en los textos tienen diversas funciones significativas: destacar, enfatizar, separar, precisar informaciones.

Además, marcan sentimientos y actitudes propios del autor o que éste quiere representar en su texto.

Los signos de puntuación más comunes son: el punto, la coma, el punto y coma, los dos puntos, el guión o raya, y los signos de admiración y de interrogación.

El punto representa el final de una unidad lingüística con sentido completo: la oración, el párrafo o el texto.

La coma representa una separación entre elementos de la misma categoría: palabras, sintagmas, frases.

El punto y coma separa formas oracionales complejas.

Los dos puntos sirven para resaltar elementos que se mencionarán a continuación.

El uso más frecuente del guión o raya es el de señalar cambio de interlocutor en el diálogo. También se usa para hacer aclaraciones dentro de una oración. 
Los signos de admiración representan sentimientos y emociones fuertes.

Los signos de interrogación indican una frase interrogativa.

Algunos ejemplos de uso no adecuado de los signos de puntuación:

Falta de comas:

"Además obtuvo su primera banda de honor la cual puso muy contento(sic) a sus padres"

Uso del punto y seguido después de una frase nominal:

"Roma caracterizada por su apodo de Tierra Santa. Mirando a su alrededor pude observar el secreto que guardan los muros..."

\subsection{Acentuaciòn}

Siempre pronunciamos en las palabras, una sílaba con más intensidad, ésta es la sílaba tónica. Pero, en la escritura no siempre marcamos esta sílaba. Para saber cuándo marcar la sílaba tónica con la tilde se deben seguir las reglas de acentuación de palabras agudas, graves y esdrújulas.

En las palabras agudas la sílaba tónica es la última y se acentúa ortográficamente cuando termina en $n, s$ o vocal.

Café, maratón, comunicación, sofá, francés...

Las palabras graves son aquellas cuya sílaba tónica es la penúltima. No se acentúan cuando terminan en $n, s$ o vocal.

Mástil, césped, lápiz, fácil...

Las palabras graves o llanas son las más frecuentes en el idioma español. 
Las palabras esdrújulas se caracterizan porque su sílaba tónica es la antepenúltima y se acentúan siempre:

Mecánico, última, gramática, frívolo, céntrico

Es común en estos días encontrar la confusión entre las palabras graves que terminan en on (llegaron) y las agudas; esto da como resultado la acentuación inadecuada de las graves, sobretodo, de los verbos conjugados en la tercera persona gramatical del plural: fuerón por fueron

Aunque en los ejemplos referidos a otros errores hemos visto también faltas en la acentuación, podemos citar algunos más:

"Regrese a mi casa inmediatamente que (sic) se acabo el cuatrimestre enero-abril 2000, pero no pude entrar al INTEC de inmediato porque debia sacar algunos documentos que demoraban (sic) mucho tiempo"

"Este no nos salió tan caro porque nos hicierón un pequeño descuento ya que heramos (sic) un grupo medio (sic) grande y cada quien iba acompañado por su pareja"

\section{RECOMENDACIONES FINALES}

Como se pudo notar en los ejemplos, los problemas gramaticales que presentan los estudiantes son abordados dentro de los contenidos de la educación formal básica. Es evidente que existe también un problema en el proceso de enseñanza aprendizaje del español en los niveles Básico y Medio de la educación dominicana.

Es dificil para el profesor de Lengua Castellana del INTEC crear una competencia gramatical en tres meses, pero se podrían adoptar algunas medidas como éstas: 
1. Identificar, a principio del trimestre, los estudiantes que presentan un mayor grado de dificultad en el dominio de los aspectos gramaticales.

2. Diseñar, con apoyo del Área de Humanidades, un programa de tutorías para los estudiantes que lo necesiten.

3. Leer en clase textos que puedan servir como ejemplos de una buena redacción.

4. Trabajar la redacción como un proceso en el que el borrador sea el texto inicial, con errores, para sucesivas revisiones y la versión final sea el producto, óptimo, de calidad que se entregue al profesor.

5. Trabajar con diferentes estructuras textuales: narraciones, noticias, biografias, textos expositivos... con las que se ejemplifiquen las características gramaticales de cada una.

6. Crear conciencia en los demás profesores del Intec acerca del problema de la escritura, del proceso de la redacción y la corrección gramatical. Esto crearía un clima en el cual la parte escrita de la lengua, con todos los aspectos que la conforman, sería usada para lograr una comunicación eficaz entre docentes y estudiantes.

\section{REFERENCIAS BIBLIOGRÁFICAS}

1. Van Dijk, Teun. Estructuras y funciones del discurso. Siglo XXI Editores, 1980. p. 59

2. (4) Van Dijk, Teun. (Comp.) El discurso como estructura y como proceso. Volumen 1. Gedisa. Barcelona. 2000. pp. 171-206

3. Jolibert Josette. Formár niños lectores de textos. Hachette. Chile. 1991.

4. Ibid 1, Pág.44

5. Ibid 1, Pág. 57

6. Ibid 1 , pág. 18 
7. Espinal, Olga. "Principales problenas en el uso de los recursos gramaticales en la producción escrita de los estudiantes del Ciclo Propedèutico del Instituto tecnológico de santo Domingo. INTEC. 2000." Ciencia y Sociedad. Santo Domingo, INTEC, vol. XXVI, No 4Octubre-Diciembre 2001.

8. Ibid 2, pàg. 177

9. Biografia. La biografia de personajes Famosos (en línea). Biografìa de Leonardo da Vinci, en:

http:/www.terra.es/personal18/biografialeonardo.htm

[Fecha de consulta: 24 de agosto de 2002]

10. Fuentes, Juan Luis. Gramática moderna de la lengua española. 14ava ed. Bibligráfica Internacional. Colombia, 1996, p. 424 